\title{
A high precision bandgap voltage reference
}

\author{
XingGuo Tian ${ }^{1, a}$, XiaoNing Xin ${ }^{2}$ and DongYang Han ${ }^{3}$ \\ ${ }^{1}$ College of Information Science and Engineering, Shenyang University of Technology, China \\ ${ }^{2}$ College of Information Science and Engineering, Shenyang University of Technology, China \\ ${ }^{3}$ College of Information Science and Engineering, Shenyang University of Technology, China
}

\begin{abstract}
In order to meet the market demand for wide temperature range and high precision bandgap voltage reference, this paper designs a bandgap reference with wide temperature range and low temperature coefficient. In this paper, the basic implementation principle of the bandgap reference is analyzed.On the basis of the traditional bandgap reference circuit structure,this design adds a trimming network and a temperature compensation network. A new Gaussian bell curve compensation technique is adopted to compensate the low temperature section, and the normal temperature section and the high temperature section respectively. Compared with the existing compensation technology, the versatility and the compensation effect is better. The designed circuit is designed and manufactured based on the Huahong HHNECGE0.35um process. The results show that the output voltage is $2.5 \mathrm{~V}$ at $2.7 \mathrm{~V}$ supply voltage and temperature range of $-40-125^{\circ} \mathrm{C}$.at typical process angle , the temperature coefficient is $0.54618 \mathrm{PPm} /{ }^{\circ} \mathrm{C}$, and is within $1 \mathrm{PPm} /{ }^{\circ} \mathrm{C}$ at other process angles.
\end{abstract}

\section{Introduction}

In recent years, with the popularity of electronic products, especially the hardware of intelligent electronic products, the power consumption and performance of chips have been put foward more and more demanding. Reference voltage sources are widely used in analog, digital and digital analog hybrid systems to provide high-precision reference voltage for IC chips. When the external environment (such as process, operating temperature, power supply voltage, load) changes, a good reference source should maintain a relatively stable state to meet the high-precision requirements of the system [1], so that the system can keep stable in the case of external environmental changes. It can be said that the quality of the voltage reference directly affects the performance of the system. When the process angle changes, the temperature coefficient of the bandgap reference source will be greatly affected. However, the current bandgap reference sources studied in many academic papers do not take into account the variation of the process angle. This paper considers the influence of the process angle and designs a Bandgap voltage reference which does not change with the process angle.

\section{Fundamental of BGR}

The basic principle of the bandgap reference is to superimpose the PTAT[1] voltage with a positive temperature coefficient and the VBE voltage with a negative temperature coefficient to achieve the purpose of reducing the temperature coefficient. The difference between the base-emitter voltages of the two bipolar transistors is the PTAT voltage. If the PTAT voltage add the VBE voltage, the output voltage $\mathrm{V}_{\mathrm{R}}$ can be written as: $V_{R}(T)=V_{B E}(T)+\alpha T \quad(1)$

Equation (1) is a generic expression, and the core part of any bandgap reference can be expressed by this formula. Where, the relationship between the second term in equation (1) and temperature is linear, and the relationship between $\mathrm{V}_{\mathrm{BE}}(\mathrm{T})$ and temperature is not linear.

$$
I_{C}(T)=I_{S}(T) \exp \left(\frac{q V_{B E}}{k T}\right)
$$

The relationship between $\mathrm{I}_{\mathrm{C}}$ and temperature is expressed by the formula (2), where the $\beta$ and $\delta$ is determined by a specific circuit.

$$
\begin{gathered}
I_{c}(T)=\beta T^{\delta}(3) \\
V_{B E}(T)=V_{G}(T)-\left(\frac{T}{T_{r}}\right) V_{G}\left(T_{r}\right)+\left(\frac{T}{T_{r}}\right) V_{B E}\left(T_{r}\right)-(\eta-\delta)\left(\frac{k T}{q}\right) \ln \left(\frac{T}{T_{r}}\right)(4)
\end{gathered}
$$

It is derived that the expression of $\mathrm{V}_{\mathrm{BE}}(\mathrm{T})$ is given by equation (3). where, $\mathrm{Tr}$ is the reference temperature, $\mathrm{T}$ is the varying temperature, $\eta$ is a process-dependent

\footnotetext{
a Xing Guo Tian: 1021134295@qq.com
} 
constant, and $\mathrm{V}_{\mathrm{G}}(\mathrm{T})$ is the relationship between the bandgap voltage of silicon with temperature.

$$
V_{B E}(T)=V_{G 0}-\frac{V_{G 0}-V_{B E}\left(T_{r}\right)}{T_{r}} T-(\eta-\delta)\left(\frac{k T}{q}\right) \ln \left(\frac{T}{T_{r}}\right)(5)
$$

Assuming that $\mathrm{V}_{\mathrm{G}}(\mathrm{T})$ is linear with temperature, the formula $(5)$ can be approximated as

$$
V_{R}(T)=V_{G 0}-(\eta-\delta)\left(\frac{k T}{q}\right) \ln \left(\frac{T}{T_{r}}\right)(6)
$$

Substituting (5) into (1) has equation (6), where in adjusting the $\alpha$ voltage cancels the primary term of $\mathrm{T}$ in equation (5), and the latter term in equation (6) is the higher order[2] term of $\mathrm{T}$, and the main component is a quadratic term after Taylor expansion. It can be seen from equation (6) that when $\eta-\delta$ is different[3], the temperature coefficient of $\mathrm{V}_{\mathrm{R}}(\mathrm{T})$ is also different, as shown in Fig. 2.1.

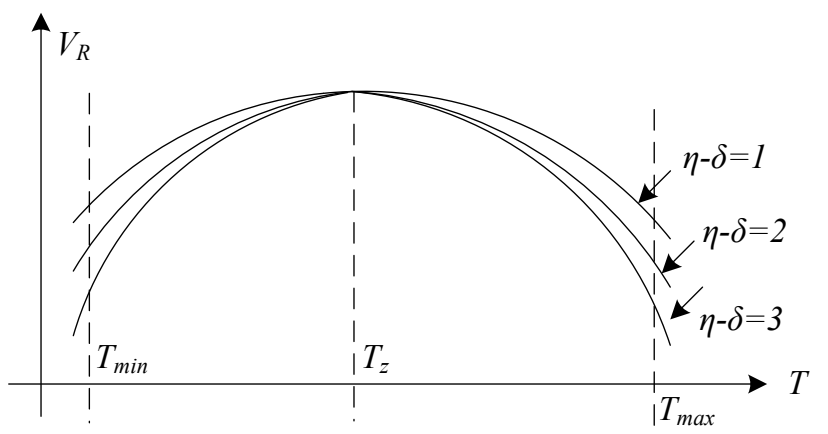

Figure 2.1. temperature characteristic between $\eta$ and $\delta$

In the literature "ASub-1-ppm/ ${ }^{\circ} \mathrm{C}$ CurvatureCompensated Bandgap Voltage Reference", the bandgap reference voltage source is curvature compensated by reducing the value of $(\eta-\delta)$. At a typical process angle, the temperature coefficient of the bandgap reference voltage source can reach $1 \mathrm{ppm} /{ }^{\circ} \mathrm{C}$, but when the process angle changes, the value of $\eta$ also changes, so the temperature coefficient in the literature will change greatly.

\section{Design of bandgap reference}

Based on the structure of the band gap reference circuit of the Brokaw $[4,5]$ structure, the design adds a trimming network, a temperature compensation[6] network in the circuit. The specific circuit design scheme is shown in Figure 3.1.and change the resistance values of R9 and R10 can adjust the output voltage zero temperature coefficient point and voltage value. Q1 is the starting circuit. The base of $\mathrm{Q} 1$ is connected to the $1.2 \mathrm{~V}$ reference voltage. When VDD is powered up, the emitter of Q1 provide an initial current for bandgap startup. After the bandgap starts, the output voltage is stable at $2.5 \mathrm{~V}$. When Q2 is turned on, Q1 will cut off. And RES3 provides the bias voltage to the temperature compensation unit. Assuming that the compensation current is ITS and IDS respectively, $\mathrm{I}_{\mathrm{TS}}$ is the compensation current of the normal temperature range, $\mathrm{I}_{\mathrm{DS}}$ is the compensation current of the low temperature section and the high temperature section, and the output voltage V2D5 can be expressed by equation (10),

$$
\begin{gathered}
I_{C 3}=I_{C 1}=\frac{V_{T} \ln 8}{R_{4}+\frac{R_{3}}{\beta}}(7) \\
V 2 D 5=\left(\frac{R_{7}}{R_{8}+R_{10}}+1\right) V_{A}+\frac{2 R_{7} I_{C 1}}{\beta}(8) \\
V_{A}=2 I_{C 3}\left(R_{6}+R_{9}+R_{5}\right)-I_{T S}\left(R_{6}+R_{9}+R_{5}\right)+I_{T D}\left(R_{6}+R_{9}\right)+V_{B E 1}(9) \\
V 2 D 5=\left(\frac{R_{3}}{R_{8}+R_{10}}+1\right)\left(\begin{array}{l}
\left(R_{6}+R_{9}+R_{5}\right) \frac{2 V_{T} \ln 8}{R_{4}+\frac{R_{3}}{\beta}} \\
-I_{T S}\left(R_{6}+R_{9}+R_{5}\right) \\
+I_{D S}\left(R_{6}+R_{9}\right)+V_{B E 1}
\end{array}\right)+\frac{2 R_{7} V_{T} \ln 8}{\beta\left(R_{4}+\frac{R_{3}}{\beta}\right)}(10)
\end{gathered}
$$

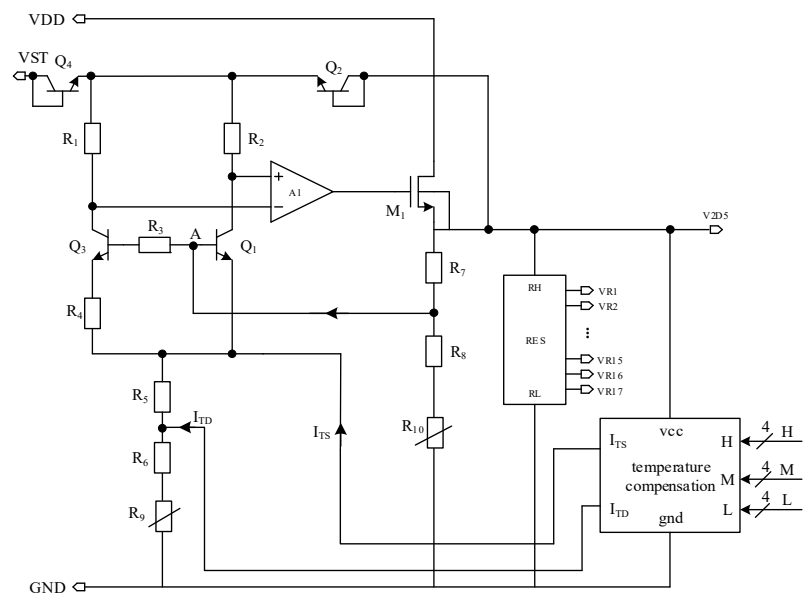

Figure 3.1. bandgap specific structure

The temperature compensation circuit in Figure 3.1, in which the compensation circuit of each temperature section uses the structure of Figure 3.3, the structure of the Gaussian current compensation unit PITC1 in Figure 3.3 use the structure of Figure 3.2, and the compensation current generated by the Gaussian bell curve has a narrow and controllable range. The low temperature section, the normal temperature section and the high temperature section can be compensated separately. The Gaussian current compensation circuit is shown in Figure 3.2. The full CMOS design is used, and the MOS operates in the subthreshold region. The expression of differential pair formed by M1 and M2 in Figure 3.2 is

$$
\begin{aligned}
& I_{D}=I_{D 0} \exp \left(\frac{V_{S G}}{\xi V_{T}}\right) \\
& V_{S G 1}=\xi_{T} \ln \left(\frac{I_{1}}{I_{D 0}}\right)(12) \\
& V_{S G 2}=\xi V_{T} \ln \left(\frac{I_{2}}{I_{D 0}}\right)(13) \\
& V_{1}-V_{2}+V_{S G 1}-V_{S G 2}=0 \\
& I_{1}+I_{2}=I_{E}(15)
\end{aligned}
$$

Substituting equation (12) for equation (13) and substituting equation (14) for equation (16), 
simultaneous equations (15) and (16) for equations (17) and (18)

$$
\begin{aligned}
& \frac{I_{1}}{I_{2}}=\exp \left(\frac{V_{2}-V_{1}}{\xi V_{T}}\right) \\
& I_{1}=\frac{I_{E}}{1+\exp \left(\frac{V_{1}-V_{2}}{\xi V_{T}}\right)}(17) \\
& I_{2}=\frac{I_{E}}{1+\exp \left(\frac{V_{2}-V_{1}}{\xi V_{T}}\right)}(18)
\end{aligned}
$$

The current mirror is used to synthesize the Gaussian bell-shaped current. The specific circuit design is shown in Figure 3.2. In the figure, M4 and M5 respectively mirror the Ia and $I_{b}$ currents. According to the linear circuit, the synthesized circuit satisfies the following relationship.

$$
I_{\mathrm{b}}=\frac{I_{E}}{1+\exp \left(\frac{V_{T}-V_{T L}}{\xi V_{T}}\right)}(19)
$$

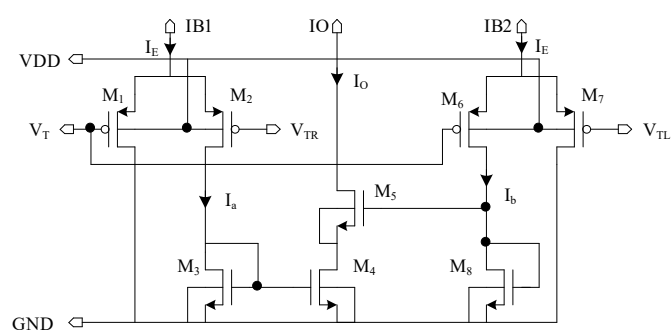

Figure 3.2. gaussian current correct unit

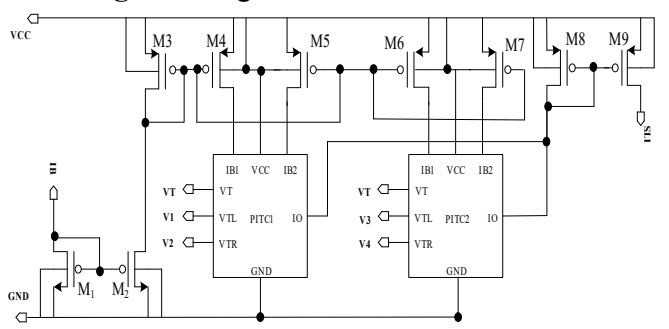

Figure 3.3. temperature compensation circuit

It can be seen from equation (20) that any curve can be spelled by changing the sizes of the $\mathrm{V}_{\mathrm{TR}}, \mathrm{V}_{\mathrm{TL}}$, and $\mathrm{I}_{\mathrm{E}}$, and does not substantially change with the change of the process angle.

\section{Results and analysis}

The circuit design, layout design, and simulation verification of this design have been completed. Figure 4.1 shows the layout design picture of the bandgap reference.

Based on the Huahong HHNEC GE0.35um process, when the process angle of MOS, resistor and bipolar transistor(abbreviated as tt-tt-tt)were changed respectively,this design used Hspice to simulate the temperature coefficient of the circuit under diffrent process angles. When the power supply voltage is $2.7 \mathrm{~V}$ and the temperature range is $-40-125^{\circ} \mathrm{C}$, the simulation results are shown in Figure 5.2. The maximum temperature coefficient under different process angles can be calculated to be $0.87288 \mathrm{PPm} /{ }^{\circ} \mathrm{C}$.

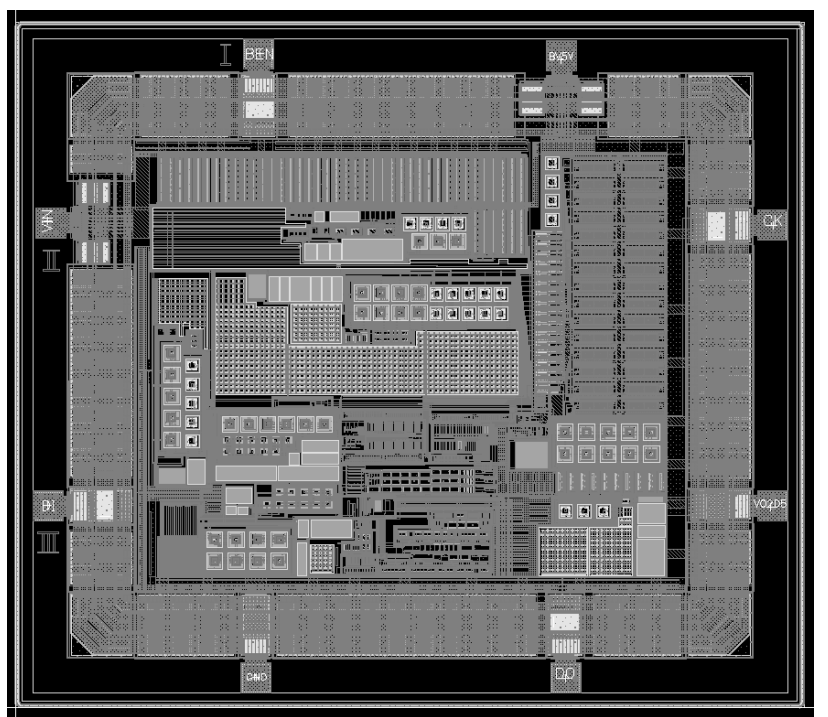

Figure 4.1. layout of bandgap vlotage reference

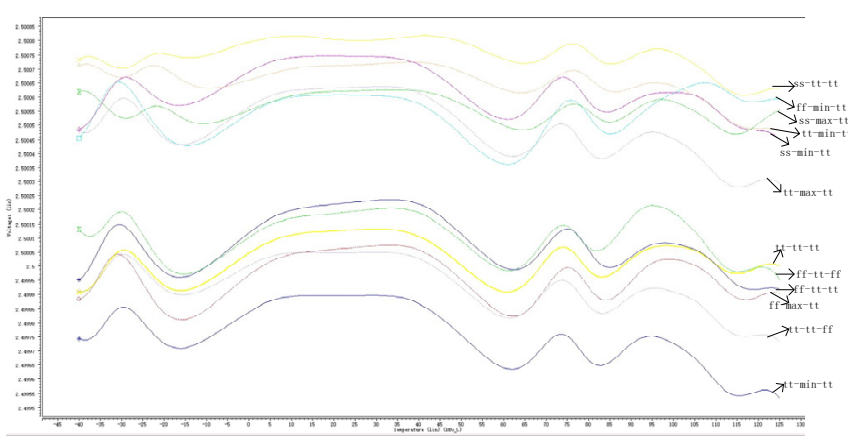

Figure 4.2. temperature characteristic curves under different process angles

\section{Conclusion}

Based on the traditional bandgap, this design adds a trimming network and a temperature compensation network to overcome the problem of the temperature coefficient of the output voltage changing with the process angle. In the temperature compensation compensation network, the controllable Gaussian bell current is innovatively proposed. The unit can make temperature compensation for each temperature range, so that the temperature coefficient under each process angle is controlled below $1 \mathrm{PPm} /{ }^{\circ} \mathrm{C}$.And the Gaussian bell shape compensation method is universal and has good compensation effect, achieving a Bandgap voltage reference with wide temperature range and low temperature coefficient. 


\section{References}

1. K. Ueno, T. Hirose, T. Asai, and Y. Amemiya, "A 1- W $600-\mathrm{ppm} /{ }^{\circ} \mathrm{C}$ current reference circuit consisting of subthreshold cmos circuits,"IEEE Trans. Circuits Syst. II, Exp. Briefs, vol. 57, no. 9, pp. 681-685,Sep. (2010)

2. G. A. Rincon-Mora, Voltage References From Diodes to PrecisionHigh-Order Bandgap Circuits. New York: IEEE Press -Wiley Interscience, pp. 65108(2002)

3. Y. H. Lam and W. H. Ki, "CMOS bandgap references with self-biased symmetrically matched current-voltage mirror and extension of sub-1-V design," IEEE Trans. Very Large Scale Integr. (VLSI) Syst.,vol. 18, no. 6, pp. 857-865, Jun. (2010)

4. K.E.Kujil. Aprecision reference voltage source.IEEE J.of solid state circuits.vol.8,pp.222226,june 1973M.

5. A. PAUL BROKAW. A Simple Three-Terminal IC Bandgap Reference. IEEE JOURNAL OF SOLIDSTATECIRCUITS,VOL.SC-9,NO. 6, DECEMBER (1974)

6. M. D. Ker and J. S. Chen, "New curvaturecompensation technique for CMOS bandgap reference with sub-1-V operation,"IEEE Trans.Circuits Syst. II, Exp. Briefs, vol. 53, no. 8, pp. 667-676, Aug. (2006) 